\title{
Automated Tracking of Pallets in Warehouses: Beacon Layout and Asymmetric Ultrasound Observation Models
}

\author{
Menasheh Fogel ${ }^{* \S}$, Nathan Burkhart ${ }^{\dagger}$, Hongliang Ren ${ }^{\ddagger}$, Jeremy Schiff ${ }^{\dagger}$, Max Meng $^{\ddagger}$, and Ken Goldberg* ${ }^{* \dagger}$
}

\begin{abstract}
We consider the use of wireless sensor networks to automatically track "perceptive pallets" of materials in warehouses for the purpose of monitoring volumetric and spatial constraints. A combination of radio frequency and ultrasound chirping produces position estimates that are noisy and prone to error. To address this, we measure and characterize the ultrasound response from standard "Cricket" wireless sensor motes and beacons. We develop a non-parametric particle filtering approach to estimate trajectories of moving motes and introduce two asymmetric observation models that incorporate measured cardioid-shaped response patterns of ultrasound. We use simulation to study the effects of mote placement: position error as a function of ceiling height and beacon density, and then perform physical experiments to evaluate the effectiveness of asymmetric vs. symmetric observation models for pallet tracking. Experiments suggest that asymmetric observation models can improve position estimates by as much as $11 \%$.
\end{abstract}

\section{INTRODUCTION}

Effectively managing materials stored in warehouses is a multi-billion dollar global challenge. Known database methods monitor entry and exit of materials, but do not monitor locations of materials. Often, materials are subject to regulatory requirements in terms of volumetric and spatial constraints. For example, US regulations for biopharma production require more than 1000 gallons of liquid corrosive (acid) material cannot be stored in one warehouse, and that an acid cannot be stored within 20 feet of a base and a flammable material cannot be stored within 20 feet of an oxidizer. Maintaining these constraints during transit and storage is a major challenge.

We consider the use of wireless sensor networks to automatically track "perceptive pallets" of materials in a warehouse to monitor such constraints. The system is required to operate dynamically in real-time, so that whenever a constraint violation is detected, the system can sound an alarm. We use simulation to study the effects of mote placement: position error as a function of ceiling height and beacon density. We then perform experiments to characterize the effectiveness of asymmetric observation models.

\footnotetext{
* Dept. of Industrial Engineering and Operations Research, University of California, Berkeley, USA

$\dagger$ Dept. of Electrical Engineering and Computer Science, University of California, Berkeley, USA

$¥$ Dept. of Electrical Engineering, Chinese University of Hong Kong, Hong Kong

$\S$ Bayer Healthcare, Pharmaceuticals Division, Berkeley, California, USA

This work was supported in part by Bayer Health Care and in part by the RGC Competitive Earmarked Research Grant CUHK415006 of the Hong Kong government, awarded to Max Meng.
}

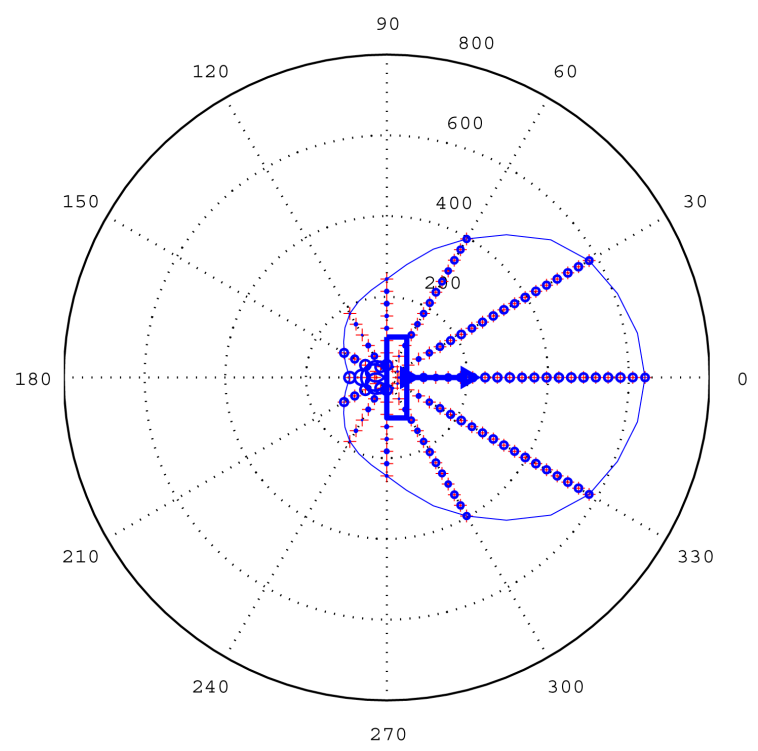

Fig. 1. Measured ultrasound response patterns overlaid with best fit asymmetric (cardioid) response models. Rectangle and arrow depict the orientation of the ultrasonic microphone. Measured position errors are asymmetric and increase with angular distance from the positive $\mathrm{x}$-axis.

Wireless sensor technologies for localization combine ultrasound bursts (chirps) with radio, infrared, or laser beams and compare time of flight to estimate distances. Currently off-the-shelf commercially available motes include Cricket [1][2], Mica2 [3], and Telos [4]. However, the distance measurements produced by these motes are noisy due to hardware limitations and environmental effects.

In our initial study [5], we evaluated the ranging capability of the Cricket Mote, and discovered that the accuracy of the sensors greatly depends on relative angle between the transmitting microphone and listening ultrasonic sensor. The experimental results of that study are illustrated in Fig. 1. In this paper, we use the measured sensitivity pattern to develop new statistical observation models and show that they reduce spatial uncertainty in position estimates. Experiments suggest that asymmetric observation models can improve position estimates by as much as $11 \%$.

\section{RELATED WORK}

\section{A. Monitoring of Spatial Constraints for Warehouse Man- agement}

Research of warehouse management has mostly focused on optimal deployment and efficient retrieval [6] according to the principles of supply chain management. One of the 
promising technologies is Radio-Frequency Identification (RFID), which has been introduced to inventory management and storage tracking [7]. Paper [8] discusses the possibility of using RFID tags to improve the localization of mobile robots, where the robot carries two overhead RFID antennas to get distance readings relative to pre-deployed RFID tags and then estimates its location from maps of the environment. An operational RFID system consists of smart-chip tags embedded with product information coupled with RFID readers. The system must be centrally operated with stateof-the-art technologies.

A similar constraint-based distance estimation method has been proposed in [9], where a spatial distance graph is constructed by labeling nodes with distance constraints. Distance intervals were derived by performing a modified Floyd-Warshall shortest path iteration algorithm for all edges. This could be a promising approach for distance violation in warehouse management, since it does not involve a complicated localization algorithm. However, perfect ranging results were assumed to implement the method, and each node must have enough memory to store the global graph and distance constraints information.

\section{B. Localization Techniques in Wireless Sensor Networks}

Localization techniques for WSNs have been extensively studied in recent years; some excellent reviews may be found in [10][11]. The most commonly used sensing approaches are acoustic, radio frequency, and laser beam. The ranging approaches are based on time of arrival (TOA), angle of arrival (AOA) [12], time difference of arrival (TDOA) [1], and received signal strength (RSS) [13]. Noisy measurements are inevitable due to the hardware constraints and environmental effects. Therefore, signal estimation techniques are proposed to infer true states from multiple sensor measurements. For instance, in [12] a sensor node localizes itself by collecting data from three or more beacon nodes.

For the particular application of spatial constraints monitoring, an inexpensive and scalable solution is required. AOA typically needs special antenna configuration and is vulnerable to multi-path reflections. RSS measurements are simple and inexpensive, but they cannot meet the dynamic resolution requirements of warehouse monitoring. Along this path, there have been some recent developments on the Mica2 mote using phase offsets of radio frequencies in [14]. Estimation error is very small ( $\sim 4 \mathrm{~cm}$ on average), but has a drawback of long convergence times (about 80 minutes) that would prohibit dynamically moving nodes. After comparing these methods, we focus on the TDOA based Cricket motes and evaluate their performance.

\section{Pros and Cons of Cricket Motes}

The Cricket location system [15] developed at MIT utilizes two types of signals, radio and ultrasound, and infers distance using the time difference of arrival of these two signals. The Cricket system implements compensation for the effects of temperature on the speed of sound, interference avoidance for arrival of radio signal and ultrasonic signals received from different transmitters, communication scheduling to avoid collision, and Kalman filtering for discarding incorrect distance samples.

In the Cricket localization algorithm, trilateration is utilized to derive the position of sensor nodes in [15], where a robust quadrilateral is introduced as a clique to avoid flip ambiguities. Additionally, signal processing in static environments using a least-square-error trilateration algorithm is used to infer position from multiple sensor readings. For a mobile environment, Kalman filters were used to derive the location. However, the selective directional sensitivity patterns of ultrasonic sensor motes make the measurements too biased, so that trilateration may be inconsistent if some of the distance readings are too noisy. Although the effects on accuracy caused by distance and angle were observed in [15], along with the existence of a cardioid sensitivity pattern, the authors did not explicitly compensate for these observations.

The most commonly recognized type of sensitivity beam patterns seen in ultrasonic sensors has one main lobe and several side lobes [16][17]. For the purposes of this paper, we abstract the sensitivity pattern to a cardioid shape without side lobes. In [18] an observation model that accounts for the sensitivity pattern of ultrasonic receivers is presented. The model incorporates angle and distance measurements; however, the sensitivity pattern does not account for received signal behind the microphone, as observed in a cardioid sensitivity pattern.

We extend the previous research on localization using Cricket motes by explicitly modeling the effect of the directional sensitivity pattern of ultrasonic sensors and making use of a probabilistic localization algorithm to derive consistent positions from noisy distance measurements.

\section{Probabilistic Localization}

Localization within a Bayesian probabilistic framework has found widespread applications in robotics [19], and has been shown to have good performance. Moreover, in some recent studies [20][21][22][10][23], methods based on a probabilistic framework have been introduced to localize within a sensor network. Probabilistic inference is made by observing multiple sets of noisy evidence. It has been shown in [11], that mobile seeds can be exploited to improve the accuracy of localization, by using Sequential Monte Carlo localization method.

Kalman filtering (KF) and Extended Kalman filtering (EKF) have been shown to be effective tools in the context of localization. However, Kalman filtering is known to be optimal only when both the transition and observation models are linear Gaussian distributions. This makes Kalman filtering infeasible for pallet monitoring, since there exist non-Gaussian components in the measurement due to the ultrasonic sensitivity pattern as illustrated in Section I. As opposed to Kalman and Extended Kalman filtering, particle filtering explicitly approximates a probability distribution as a sample set. The advantage of particle filtering is its 
capability to represent an arbitrary probability model and its flexibility in dealing with dynamic and distributed systems.

Particle filtering is an implementation of Bayesian filtering based on a Sequential Monte Carlo (SMC) method [24]. The features of the method are a probabilistic transition model between each state and a probabilistic observation model to estimate state by observed evidence. The key of particle filtering is the representation of the posterior distribution by a set of weighted samples (or particles), so that it can approximately represent any probability distribution. In [20], a particle filtering approach is used to track intruders by a number of infrared sensors with binary outputs. The authors equipped the room with an array of binary sensors, characterized the firing probability and refractory period of these sensors by conditional probability distributions (CPDF), and derived the posterior probability by observing the firing events.

It has been shown that the accuracy of particle filtering is related to the number of samples. Therefore, the computation complexity of particle filter is directly related to the number of samples, and the optimal number of particles is difficult to determine. A computational complexity analysis of particle filters can be found in [25]. In our experience, however, we have found the trade-off between these convergence times and accuracy to be more than acceptable for the proposed application.

\section{Problem Statement}

The objective of our system is to monitor the location of Cricket sensor motes in a room of known dimensions. This tracking must operate in real time, and it must accurately localize both mobile and stationary sensors. In our proposed global architecture, envisioned in [5], Cricket motes (referred to as Beacons) are statically mounted in known locations on the ceiling, facing downward. Cricket motes (referred to as Motes) are also affixed to pallets placed on the floor, facing upward. Refer to Fig. 2 for a visual depiction of the described setup.

Each Mote broadcasts both an ultrasound pulse and a radio message containing a unique Mote identifier and a monotonically increasing message number at a pre-determined rate managed by an internal timer. The message number is used by the Beacons to differentiate between messages and thus no global synchronization is required. Every Beacon that receives a radio message and ultrasound pulse computes its estimated distance from the Mote based on the difference in arrival time of the two signals. The distance estimates computed by all Beacons are centrally aggregated, and our algorithm uses these estimates to localize the Motes in discrete time.

\section{A. Assumptions}

It is assumed that the floor and ceiling are both flat and relatively level, and that all pallets are flat and of a uniform height. Other than the Beacons, Motes, and pallets, there are no other objects in the room. There is a central computer that is able to communicate directly with each

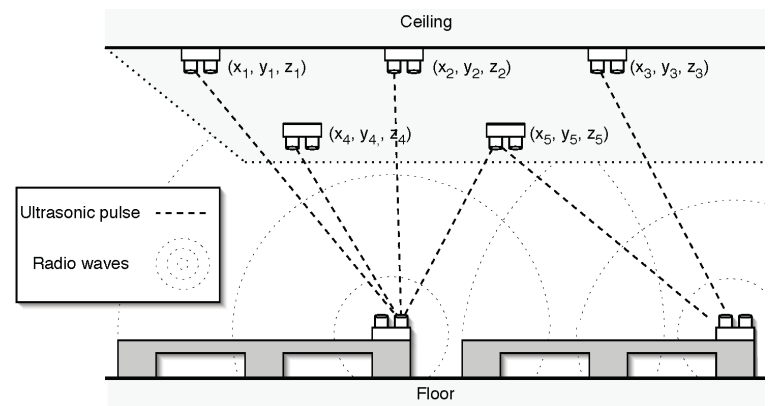

Fig. 2. Depiction of layout of sensors for monitoring the location of hazardous materials in warehouses. The Cricket motes on the ceiling are referred to as Beacons, while the Cricket motes affixed to the pallets on the floor are referred to as Motes.

ceiling-mounted Beacon; this is where the algorithm is run. It is also assumed that all pairwise distance estimates are probabilistically independent of each other.

\section{B. System Input}

The system is initialized with the Euclidean coordinates of each Beacon. The dimensions of the room are also specified by length, width, and height. The system collects pairwise distance estimates between some subset of all existing $\{$ Beacon, Mote $\}$ pairs at a predefined rate.

\section{System Output}

The system computes the estimated Euclidean coordinates of each Mote, with the objective of minimizing error defined as Euclidean distance between estimated and true position.

\section{USING PARTICLE FILTERING FOR LOCALIZATION}

\section{A. Overview of Particle Filtering}

In this subsection, we present a generalized model and notation for particle filtering. Our system employs the Sampling Importance Resample (SIR) Particle Filter described in [26]. It is a method used for performing state estimation of Dynamic Bayes Nets over discrete time. The state of the object at time $t$ is represented by the random variable $x_{t}$, and the observations of the object's actual state collected at time $t$ are represented by the random variable $z_{t}$. There are three distributions used in the algorithm: the distribution of the object's initial state, $\mathrm{P}\left(x_{0}\right)$, the transition model, $\mathrm{P}\left(x_{t} \mid x_{t-1}\right)$, and the observation model, $\mathrm{P}\left(z_{t} \mid x_{t}\right)$. A collection of samples (also referred to as particles) of the object's state is maintained, distributed according to the likelihood of all past observations: $\mathrm{P}\left(x_{t} \mid z_{0: t}\right)$.

At each iteration, every sample state is advanced according to the transition model. It is then assigned a probability according to its likelihood using the defined observation model. These probabilities are normalized, and the set of samples is then resampled with replacement according to the computed relative probabilities. This new distribution of samples implicitly integrates all previous observations, as a function of the algorithm, and they are distributed according to their likelihood. The number of sample states corresponding to a particular state is proportional to the likelihood that the state matches that of the object. 


\section{B. Particle Filtering Applied to Localization}

Table I contains important notation that will be used in the following sections. In our framing of the localization problem, we run a separate particle filter for each individual Mote. An object's state at time $t, x_{i t}$, refers to the true Euclidean coordinates of Mote $i$. The observations collected at time $t, \mathbf{z}_{\mathbf{i t}}$, refer to a vector of distance estimates computed by the Beacons between time $t-1$ and time $t$, relative to Mote $i$. Our transition model, $\mathrm{P}\left(\hat{x}_{i s t} \mid \hat{x}_{i s(t-1)}\right)$, simply adds Gaussian noise to the Euclidean coordinates of $\hat{x}_{i s(t-1)}$. The initial distribution of samples, $\mathrm{P}\left(\hat{x}_{i s 0}\right)$, places sample states uniformly at random within the defined space. Our observation model, $\mathrm{P}\left(\mathbf{z}_{\mathbf{i t}} \mid \hat{x}_{i s t}\right)$, gives the likelihood of the computed distance estimates based on empirical data gathered about the sensitivity pattern of the Cricket motes. A visual depiction of the algorithm can be seen in Fig. 3 .

TABLE I

NOTATION

\begin{tabular}{|c|c|}
\hline$M$ & The set of Motes, $i \in M$. \\
\hline$B$ & The set of Beacons, $j \in B$. \\
\hline$S$ & The set of Samples, $s \in S$. \\
\hline$T$ & The set of particle filter iteration Times, $t \in T$. \\
\hline$h$ & $\begin{array}{l}\text { The height of a mounted beacon, assumed to be a } \\
\text { uniform constant. }\end{array}$ \\
\hline$x_{i t}$ & $\begin{array}{l}\text { The true state of Mote } i \text { at time } t . \text { State is defined } \\
\text { by Euclidean }(x, y) \text { coordinates. }\end{array}$ \\
\hline$z_{i j}$ & $\begin{array}{l}\text { The distance estimate between Mote } i \text { and Beacon } j \\
\text { during calibration. }\end{array}$ \\
\hline$d_{i j}$ & $\begin{array}{l}\text { The true Euclidean distance between Mote } i \text { and } \\
\text { Beacon } j \text { during calibration. }\end{array}$ \\
\hline$\theta_{i j}$ & $\begin{array}{l}\text { The true relative angle between Mote } i \text { and Beacon } \\
j \text { during calibration. }\end{array}$ \\
\hline$z_{i t}$ & $\begin{array}{l}\text { The vector }\left[z_{i 1 t}, \ldots, z_{i j t}, \ldots, z_{i m t}\right] \text { of computed } \\
\text { distance estimates relative to Mote } i \text { at time } t \text {, } \\
\text { relative to each Beacon } j \text {, where } m \text { is the number } \\
\text { of Beacons. }\end{array}$ \\
\hline$\hat{x}_{i t}$ & The estimated state of Mote $i$ at time $t$. \\
\hline$\hat{x}_{i s t}$ & $\begin{array}{l}\text { The state of sample } s \text { representing a possible state } \\
\text { of Mote } i \text { at time } t \text {. }\end{array}$ \\
\hline$\hat{d}_{i j s t}$ & $\begin{array}{l}\text { The Euclidean distance between the sample state } \\
\hat{x}_{i s t} \text { and Beacon } j \text {. }\end{array}$ \\
\hline$\hat{\theta}_{i j s t}$ & $\begin{array}{l}\text { The relative angle between the sample state } \hat{x}_{i s t} \text { and } \\
\text { Beacon } j \text {. }\end{array}$ \\
\hline$a, b, c, p, q, r$ & Coefficients used for experimental data fitting. \\
\hline
\end{tabular}

\section{Deriving the Observation Model}

It was demonstrated in [5] that the pairwise distance estimates produced by Cricket motes vary based on the relative angle of the two motes. By the independence assumption of distance estimates, the observation model is expressed as the following:

$$
\mathrm{P}\left(\mathbf{z}_{\mathbf{i t}} \mid \hat{x}_{i s t}\right)=\prod_{j=1}^{m} \mathrm{P}\left(z_{i j t} \mid \hat{x}_{i s t}\right)
$$

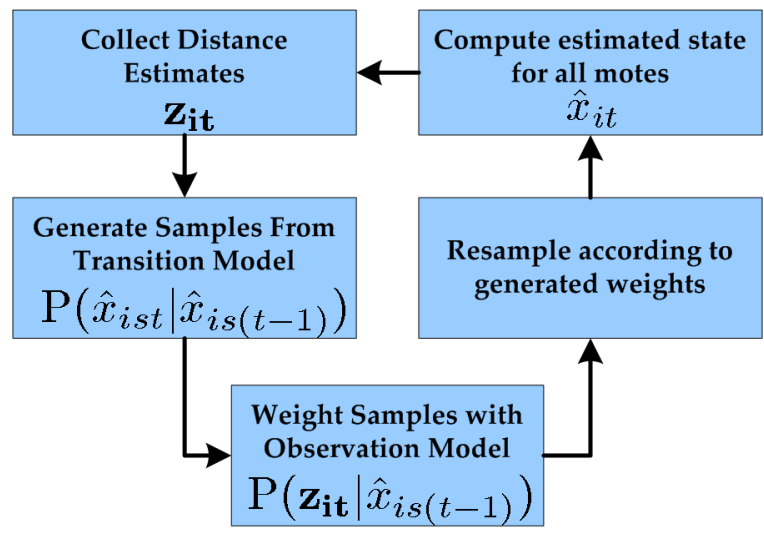

Fig. 3. An overview of one iteration $t$ of particle filtering for localization. Pairwise distance estimates are collected $\mathbf{z}_{\mathbf{i t}}$. Samples are generated by the transition model $\mathrm{P}\left(\hat{x}_{i s t} \mid \hat{x}_{i s(t-1)}\right)$. The observation model then assigns each sample a weight according to $\mathrm{P}\left(\mathbf{z}_{\text {it }} \mid \hat{x}_{i s t}\right)$, and the particles are resampled using these weights. The output is the mote's estimated state $\hat{x}_{i t}$.

Because the locations of all Beacons $B$ are known, given a sample state $\hat{x}_{i s t}$ we can compute the Euclidean distance and relative angle to each individual Beacon $j$. Let $\left(x_{i}, y_{i}\right)$ represent the state $\hat{x}_{i s t}$, let $\left(x_{j}, y_{j}\right)$ represent the Euclidean coordinates of Beacon $j$, and let $h$ represent the height of the ceiling to which $j$ is mounted. For each pair $\left\{\hat{x}_{i s t}, j\right\}$, we can compute $\hat{d}_{i j s t}$ and $\hat{\theta}_{i j s t}$ as follows:

$$
\begin{aligned}
& \hat{d}_{i j s t}=\sqrt{\left(x_{i}-x_{j}\right)^{2}+\left(y_{i}-y_{j}\right)^{2}+h^{2}} \\
& \hat{\theta}_{i j s t}=\arccos \left(\frac{h}{\hat{d}_{i j s t}}\right)
\end{aligned}
$$

Using these two aspects of a Mote's state, we can now express our observation model as the following:

$$
\mathrm{P}\left(\mathbf{z}_{\mathbf{i t}} \mid \hat{x}_{i s t}\right)=\prod_{j=1}^{m} \mathrm{P}\left(z_{i j t} \mid \hat{d}_{i j s t}, \hat{\theta}_{i j s t}\right)
$$

\section{Using Experimental Data to Model Acoustic Sensitivity}

During the calibration phase, we fit the observation model to the underlying truth from experimental data. Calibration is performed once before the particle filtering algorithm is executed. From the results in [5], we can examine the distance estimate $z_{i j}$ produced between a Mote $i$ and Beacon $j$ based on their true relative distance $d_{i j}$ and angle $\theta_{i j}$. In these experiments, the distance estimates $z_{i j}$ were collected for a variety of $\left\{d_{i j}, \theta_{i j}\right\}$ pairs.

Because the acoustic sensitivity pattern of the ultrasound signal exhibits a cardioid shape, as depicted in Fig 1, we assume that the mean, $\mu$, and the variance, $\sigma^{2}$ can be reasonably approximated as a polar function of any arbitrary distance and angle pair $\{d, \theta\}$. We refer to this observation model as Asymmetric Polar (AP):

$$
\begin{aligned}
\mu(d, \theta) & =a d \cos \theta+b d \sin \theta+c \\
\sigma^{2}(d, \theta) & =p d \cos \theta+q d \sin \theta+r
\end{aligned}
$$


Using linear regression, we computed the specific values for the coefficients $a, b, c, p, q, r$ that provided the least squared error over all pairs $\left\{d_{i j}, \theta_{i j}\right\}$ for which there was available experimental data. By preprocessing these coefficients, much of the computation is implicitly performed by the formulation, rather than at run time. Given these coefficients, we can compute $\mu(d, \theta)$ and $\sigma^{2}(d, \theta)$ for any $\{d, \theta\}$. These $\mu$ and $\sigma^{2}$ functions can then be substituted into the standard Gaussian probability density function. Thus, the probability distribution given in Eq. 4 can be approximated using the following function, instantiated with actual $\left\{\hat{d}_{i j s t}, \hat{\theta}_{i j s t}\right\}$ values:

$$
\begin{aligned}
& \mathrm{P}\left(z_{i j t} \mid \hat{d}_{i j s t}, \hat{\theta}_{i j s t}\right)= \\
& \frac{1}{\sqrt{2 \pi \sigma^{2}\left(\hat{d}_{i j s t}, \hat{\theta}_{i j s t}\right)}} \exp \left\{-\frac{\left(\mu\left(\hat{d}_{i j s t}, \hat{\theta}_{i j s t}\right)-z_{i t j}\right)^{2}}{2 \sigma^{2}\left(\hat{d}_{i j s t}, \hat{\theta}_{i j s t}\right)}\right\}
\end{aligned}
$$

To compare the formulation as a polar function, we constructed a similar observation model with $\mu$, and $\sigma^{2}$ formulated as linear functions of $d$ and $\theta$. This observation model is referred to as Asymmetric Linear (AL). For this model, Eqns. 5 and 6 are formulated as follows:

$$
\begin{aligned}
\mu(d, \theta) & =a d+b \theta+c \\
\sigma^{2}(d, \theta) & =p d+q \theta+r
\end{aligned}
$$

We then computed this set of coefficients $a, b, c, p, q, r$ using the same approach as described above. Using these coefficients and the functions in Eqns. 8 and $9, \mu$, and $\sigma^{2}$ can be used in Eq. 7 without additional modification.

Finally, to test the effect of the relative angle of mote poses when computing distance estimates, we also constructed an observation model with $\mu$, and $\sigma^{2}$ formulated as linear functions of only distance $d$. This observation model is referred to as Symmetric Linear (SL). For this model, Eqns. 5, and 6 are formulated as follows:

$$
\begin{aligned}
\mu(d, \theta) & =a d+0 \theta+c \\
\sigma^{2}(d, \theta) & =p d+0 \theta+r
\end{aligned}
$$

Again, this set of coefficients $a, b, c, p, q, r$ was computed using linear regression with $b=q=0$. Using these coefficients and the functions in Eqns. 10 and 11, $\mu$, and $\sigma^{2}$ can be used in Eq. 7 without additional modification. The accuracy with which each of these models localized the Motes is evaluated in Section V-B.

\section{EXPERIMENTAL RESULTS}

We evaluate the performance of our algorithm with simulator and physical experiments. The objective of the simulator experiments is to determine an acceptable physical beacon configuration. To determine which configurations are acceptable, we look for a layout suitable for a warehouse environment that has estimation error less than $10 \mathrm{~cm}$. We define estimation error $\left(\left|x_{i t}-\hat{x}_{i t}\right|, \forall i \in M, t \in T\right)$ as an objective in Section III-C.

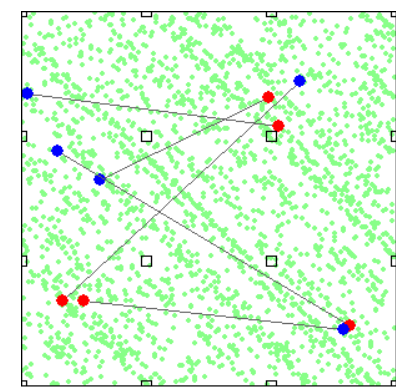

(a) Initial Distribution

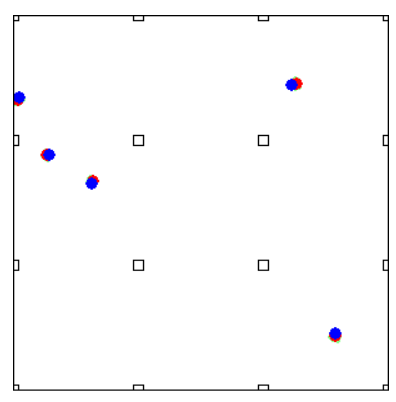

(b) After Convergence
Fig. 4. Graphical User Interface of Particle filter simulator. (a) Initial particle distribution of first iteration. (b) Estimated positions from of the motes. The blue dots represent the actual poses of motes, red dots for the estimated ones, squares for beacons and green dots for particles. Though difficult to see, particles are present under the estimated positions in (b). The length of the line connecting the actual motes and estimated nodes reflect the errors of estimation.

\section{A. Simulation Results}

To describe the best physical configuration of beacons, we visualize estimation error according to the dimensions of ceiling height and beacon density. We constructed a simulator that was capable of performing many trials in a short amount of time. Performance was measured as distance estimation error for various ceiling heights $h$ (reported in $\mathrm{cm}$ ) and inter-beacon (beacon to beacon) distances $k$ (reported in $\mathrm{cm}$ ). Beacons are evenly spaced at the inter-beacon distance in a grid. Our objective was to determine an appropriate beacon configuration in order to minimize total computation expense, total number of needed beacons at a reasonable ceiling height while still maintaining an acceptable measure of error.

The simulator is implemented in a Java 1.5 environment with an Applet Graphical User Interface (GUI) used to visualize the actual positions, estimated positions, and particle locations of Beacons and Motes (shown in Fig. 4). At initialization, a set of particles for each node is generated with a uniform distribution as shown in Fig. 4(a). After computing the observed measurements, the posterior probability is calculated and the particles converge to an estimated position as shown in Fig. 4(b). Each mote is given a random trajectory of $\left(x_{t}, y_{t}\right)$ pairs. The trajectory is generated at each time step by perturbing the current, true position with a sample drawn from a Gaussian distribution with $(\mu=0, \sigma=10)$.

The observation model used for the experiments is Asymmetric Linear. Experiments were run on IBM xSeries 330 machines equipped $1.0 \mathrm{GHz}$ Intel Pentium III CPUs and 1.5GB ECC PC133 SDRAM. Each experiment consisted of 10 motes, over 10 iterations, with 5000 particles per mote. These experiments had an average run time of 1.25 minutes.

For our first experiment, we evaluate ceiling height with moving nodes. Ten motes are placed in the simulator space of $1000 \times 1000 \mathrm{~cm}$ size room. Beacon density is held constant and ceiling height is varied from $h=120, \ldots, 640$ with a linear step size of 10 . This measurement is evaluated for 


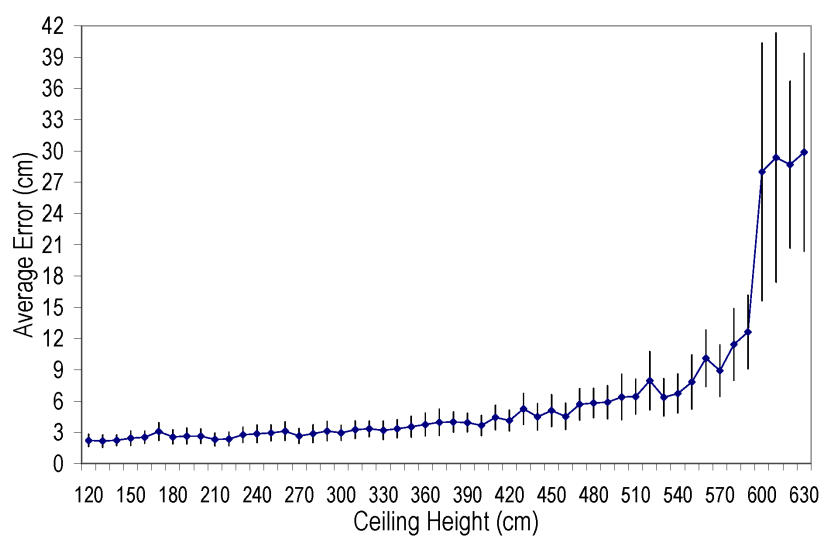

Fig. 5. Estimation error against ceiling height. In this representative plot, inter-beacon distance $k=180$ and particle density is 5000 . Average error of all 10 nodes over 10 iterations is shown against each ceiling height. Standard deviation is shown with vertical bars. A sharp threshold is shown for $h>590$.

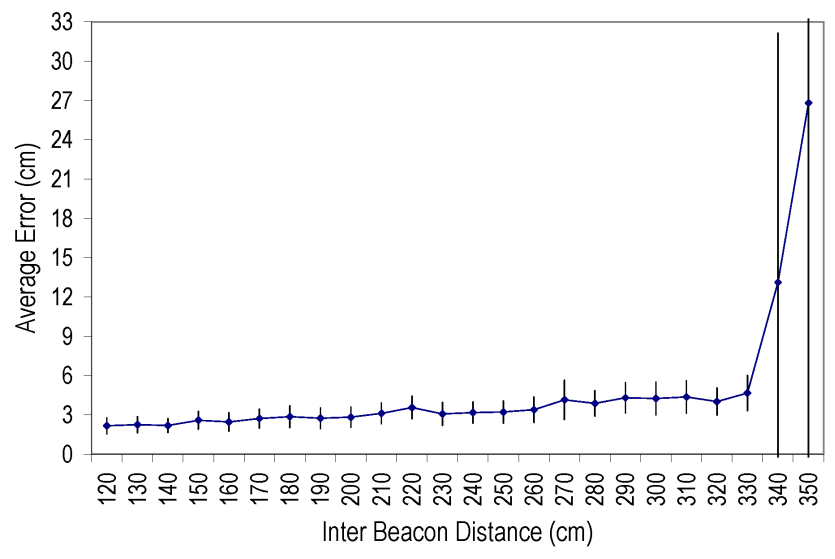

Fig. 6. Estimation error against beacon density. In this representative plot, ceiling height $h=240$ and particle density is 5000. Average error of all 10 nodes over 10 iterations is shown against each inter-beacon distance. Standard deviation is shown with vertical bars. A sharp threshold is shown for $k>330$.

all beacon densities as described in the next experiment. As shown in Fig. 5, distance estimation error increases proportionally with ceiling height and is sharply thresholded for heights above $590 \mathrm{~cm}$.

In the next experiment, we evaluate beacon density with moving nodes. Again, ten motes are placed in a simulator space of an 1000x1000 cm size room. Ceiling height is held constant while inter-beacon distance is varied from $k=$ $120, \ldots, 350$ with a linear step size of 10 . This measurement is evaluated for all ceiling heights evaluated in the previous experiment. As shown in Fig. 6, distance estimation error increases proportionally with inter-beacon distance and is sharply thresholded for distances above $330 \mathrm{~cm}$.

Both ceiling height and inter-beacon density are plotted in Fig. 7. This plot depicts an "acceptable zone" of interbeacon density and ceiling height. We define acceptable to be configurations where the error is less than $10 \mathrm{~cm}$.

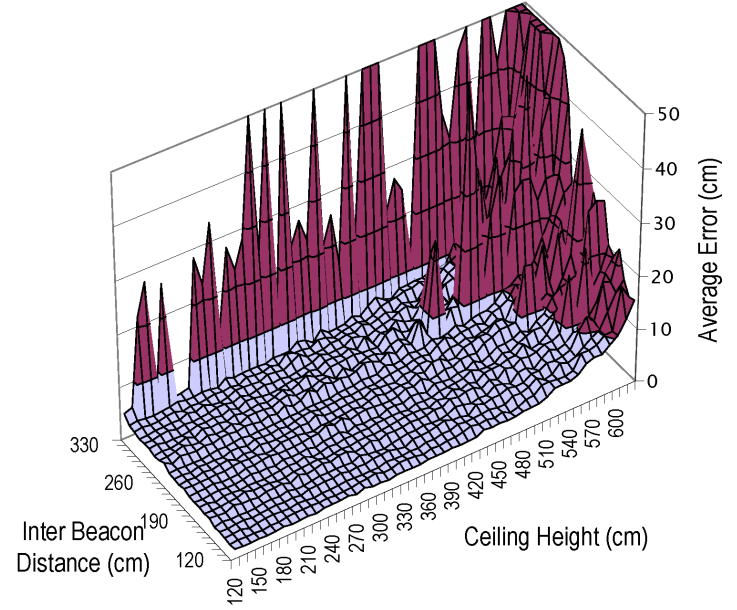

Fig. 7. Estimation error along both dimensions studied for beacon configuration. This plot depicts an "acceptable zone" for beacon configurations. Any configuration with error less than $10 \mathrm{~cm}$ is deemed acceptable.

\section{B. Physical Experiment Results}

Using the configuration results of the simulator, we installed the motes in our lab to test performance. The model of the mote was MCS410CA (or Cricket mote v2), running TinyOS 1.1.7. Using the MIB510CA programming board, the Cricket software (release 2.3.0) was modified to transmit pairwise distances to a bay station. None of the on-board distance calculations were modified.

The primary metric of our experiment was again distance estimation error $\left|x_{i t}-\hat{x}_{i t}\right|, \forall i \in M, t \in T$. We installed six Cricket motes in beacon mode at a uniform height of 274 $\mathrm{cm}$ with an approximate inter-beacon distance of $198 \mathrm{~cm}$. Beacons were installed parallel to the floor with ultrasonic sensors directed downward. Rotation parallel to the floor plane is not explicitly controlled, whereas tilt and yaw are avoided. A trajectory was then marked on the floor with the same $\mathrm{x}$-y coordinate plane as the beacons. One cricket was configured as a listener and then moved along the trajectory on the floor.

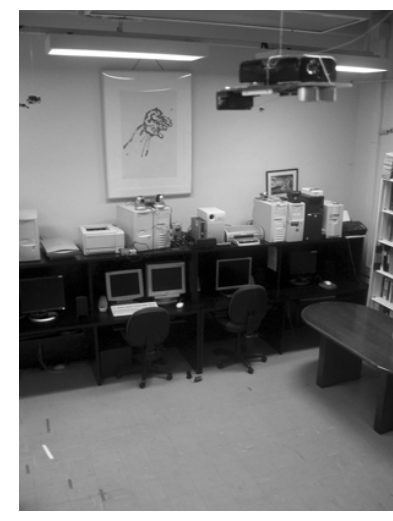

(a) Beacon installation

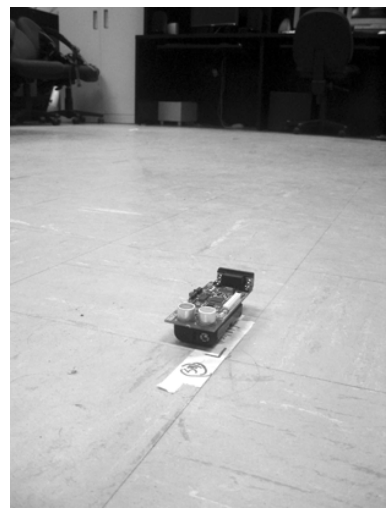

(b) Tracked Mote
Fig. 8. Installed cricket motes at a uniform height of $274 \mathrm{~cm}$ (a). Ultrasonic sensors are directed downward. Trajectory of the mote as steps are marked on the floor and measured to get the actual location (b). Mote to be localized is directed upward. 


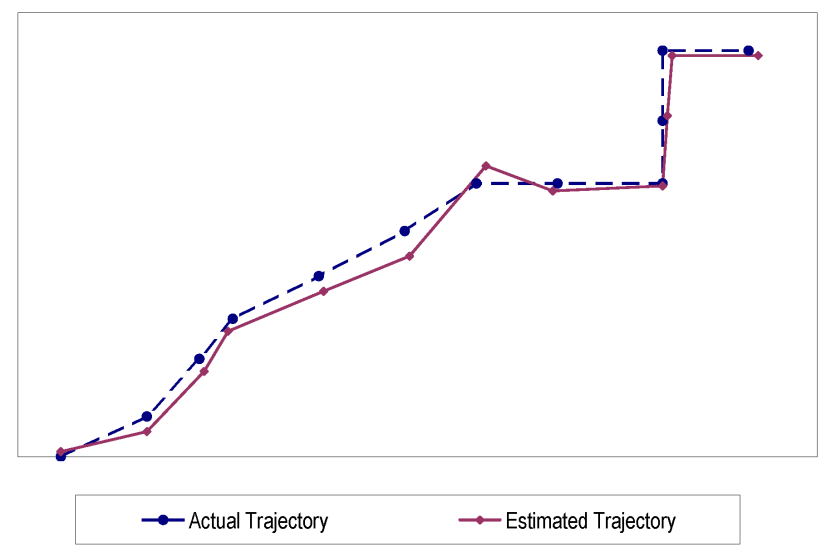

Fig. 9. Top view of the lab floor. Lines indicate the actual versus estimated trajectory in a portion of the lab of $450 \mathrm{~cm} \mathrm{x} 250 \mathrm{~cm}$. The observation model for this experiment is Asymmetric Polar. The plot depicts one experimental run whose overall estimation error was closest to the mean reported for the AP observation model.

In order to apply our algorithm to the sensor evidence, the motes were programmed to send pairwise distance estimates at a set interval to the bay station. The experiment is designed to control specific locations at specific iterations. Accordingly, each iteration is 10 seconds long. To process the algorithm, the recorded pairwise distances are passed into the simulator to analyze and visualize the estimated positions of the motes. A comparison of actual and estimated trajectory of the experimental run is shown in Fig. 9.

We compare the effect on estimation error for the three observation models Asymmetric Polar (AP), Asymmetric Linear (AL), and Symmetric Linear (SL). Using the same experimental data from the physical experiments, the three observation models are compared in Fig. 10. It is shown that an asymmetric observation model (AL and AP) is strictly better than an observation model that does not consider angle (SL). Moreover, this experiment demonstrates that a linear approximation with angular dependence of the empirical data of the ultrasonic sensors (AL) has performance that is very close to that of an approximation that considers the polar model of the cardioid sensitivity pattern (AP). The estimation error for each model over all physical experiments is SL: $\mu=4.40, \sigma=4.50, \quad \mathrm{AL}: \mu=4.01, \sigma=4.06$, and AP: $\mu=3.91, \sigma=4.05$. These experiments suggest that asymmetric observation models can improve position estimates by as much as $11 \%$.

\section{CONCLUSION AND FUtURE WORK}

We consider the use of wireless sensor networks to automatically track "perceptive pallets" of materials in warehouses for the purpose of monitoring volumetric and spatial constraints. We measure and characterize the ultrasound response from standard "Cricket" wireless sensor motes and beacons. We develop a non-parametric particle filtering approach to estimate trajectories of moving motes and introduce two asymmetric observation models that incorporate

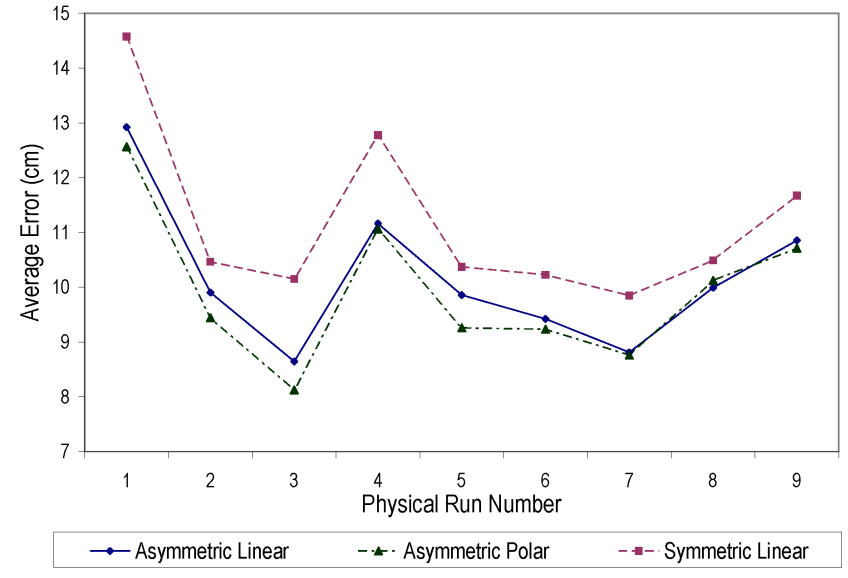

Fig. 10. Comparison of the three observation models in this paper. The pairwise distances of 33 iterations from the physical experiment are compared over 9 experimental runs of 50 particle filter estimations each. When angle is considered (AL and AP), error is shown to be strictly less than a distance only observation model.

measured cardioid-shaped response patterns of ultrasound. We use simulation to study the effects of mote placement: position error as a function of ceiling height and beacon density, and then perform physical experiments to evaluate the effectiveness of asymmetric vs. symmetric observation models for pallet tracking.

The key contribution of our research is to measure the effects of spatial asymmetry in ultrasonic sensors and to incorporate these sensitivity patterns into the particle filtering observation model.

In future work, we will incorporate the fact that we usually have a precise map of the warehouse interior. Since ultrasound requires line of sight communication, this map can account for some of these challenges by explicitly considering known obstacles, known transportation routes, and known storage areas. We will combine this information with the particle filter to further improve position estimates and develop fast algorithms for automatic monitoring of volumetric and spatial constraints. Additionally, we will expand the physical experimentation to an actual warehouse environment using the techniques and configurations recommended by this research.

\section{ACKNOWLEDGMENT}

The authors gratefully acknowledge Jörg Heidrich, Edgar Sur and Prasad Saraph for enabling the collaboration between Bayer HealthCare and Goldberg's Automation Sciences Lab at UC Berkeley. We also thank Profs. George Shanthikumar and Mike Franklin for inspiring ideas and initial work. Additionally, we thank Mr. Philippe Cudré-Mauroux, School of Computer and Communication Sciences, EPFL, Switzerland, and Laura Pedretti, for graphical expertise.

\section{REFERENCES}

[1] D. Moore, J. Leonard, D. Rus, and S. Teller, "Robust distributed network localization with noisy range measurements," in 2nd interna- 
tional conference on Embedded networked sensor systems, Baltimore, Maryland, USA, Nov. 2004, pp. 50-61.

[2] N. Priyantha, A. K. Miu, H. Balakrishnan, and S. Teller, "The cricket compass for context-aware mobile applications," in 7th annиal international conference on Mobile computing and networking, Rome, Italy, Jul. 2001, pp. 1-14

[3] J. L. Hill and D. E. Culler, "Mica: a wireless platform for deeply embedded networks," Micro, IEEE, vol. 22, no. 6, pp. 12-24, 2002

[4] J. Polastare, R. Szewczyk, and D. Culler, "Telos: enabling ultralow power wireless research," in 4th international symposium on Information processing in sensor networks, Los Angeles, California, USA, Apr. 2005, p. 48.

[5] P. Cudre-Mauroux, M. Fogel, K. Goldberg, and M. Franklin, "Sentry pallets for automated monitoring of spatial constraints: Models and initial experiments," in IEEE International Conference on Robotics and Automation (ICRA'O6), Orlando, Florida, USA, May 2006, pp. 4396-4398.

[6] J. P. V. den Berg, "A literature survey on planning and control of warehousing systems," IIE Transactions, vol. 31, no. 8, pp. 751-762, 1999.

[7] K. Michael and L. McCathie, "The pros and cons of rfid in supply chain management," in International Conference on Mobile Business (ICMB '05), Sydney, Australia, Jul. 2005, pp. 623-629.

[8] D. Hahnel, W. Burgard, D. Fox, K. Fishkin, and M. Philipose, "Mapping and localization with rfid technology," in IEEE International Conference on Robotics and Automation (ICRA '04), Boston, Massachusetts, USA, Apr. 2004, pp. 190-202.

[9] U. Bischoff, M. Strohbach, M. Hazas, and G. Kortuem, "Constraintbased distance estimation in ad-hoc wireless sensor networks," in $3 r d$ European Workshop on Wireless Sensor Networks (EWSN '06), Zurich, Switzerland, Feb. 2006, pp. 54-68.

[10] K. Langendoen and N. Reijers, "Distributed localization in wireless sensor networks: a quantitative comparison," Comput. Networks, vol. 43, no. 4, pp. 499-518, 2003.

[11] L. Hu and D. Evans, "Localization for mobile sensor networks," in 10th annual international conference on Mobile computing and networking, Philadelphia, Pennsylvania, USA, Sep. 2004, pp. 45-57.

[12] A. Nasipuri and K. Li, "A directionality based location discovery scheme for wireless sensor networks," in 1st ACM international workshop on Wireless sensor networks and applications, Atlanta, Georgia, USA, Sep. 2002, pp. 105-111.

[13] N. Patwari and A. O. H. III, "Using proximity and quantized rss for sensor localization in wireless networks," in 2nd ACM international conference on Wireless sensor networks and applications, San Diego, California, USA, Sep. 2003, pp. 20-29.

[14] M. Maroti, B. Kusy, G. Balogh, P. Volgyesi, K. Molnar, A. Nadas, S. Dora, and A. Ledeczi, "Radio interferometric positioning," Institute for Software Integrated Systems, Vanderbilt University, TN, Tech. Rep. ISIS-05-602, Nov. 2005

[15] N. B. Priyantha, "The cricket indoor location system," Ph.D. dissertation, Massachusetts Institute of Technology, Cambridge, Massachusetts, USA, Jun. 2005.

[16] H. Choset, K. Nagatani, and N. A. Lazar, "The arc-transversal median algorithm: a geometric approach to increasing ultrasonic sensor azimuth accuracy," IEEE Transactions on Robotics and Automation, vol. 19, no. 3, pp. 513-521, 2003.

[17] D. P. Massa, "Choosing an ultrasonic sensor for proximity or distance measurement; part 2: Optimizing sensor selection," Sensors, vol. 16, no. 3, pp. 28-43, 1999.

[18] B. Barshan and R. Kuc, "A bat-like sonar system for obstacle localization," IEEE Transactions on Systems, Man, and Cybernetics, vol. 22, no. 4, pp. 636-646, Jul. 1992.

[19] S. Thrun, "Probabilistic robotics," Commun. ACM, vol. 45, no. 3, pp. $52-57,2002$

[20] J. Schiff and K. Goldberg, "Automated intruder tracking using particle filtering and a network of binary motion sensors," in IEEE International Conference on Automation Science and Engineering (CASE'06), Shanghai, China, Oct. 2006, pp. 1-2.

[21] R. Stoleru, T. He, J. A. Stankovic, and D. Luebke, "A high-accuracy, low-cost localization system for wireless sensor networks," in $3 \mathrm{rd}$ international conference on Embedded networked sensor systems, San Diego, California, Nov. 2005, pp. 13-26.

[22] C. Taylor, A. Rahimi, J. Bachrach, H. Shrobe, and A. Grue, "Simultaneous localization, calibration, and tracking in an ad hoc sensor network," in 5th International Conference on Information Processing in Sensor Networks, Nashville,Tennessee,USA, Apr. 2006, pp. 27-33.

[23] H. Durrant-Whyte and T. Bailey, "Simultaneous localization and mapping: Part i," IEEE Robotics and Automation Magazine, vol. 13 , no. 2, pp. 99-110, Jun. 1996.

[24] S. Thrun, W. Burgard, and D. Fox, Probabilistic Robotics. Cambridge, Massachusetts: MIT Press, 2005.

[25] M. Bolic, P. M. Djuric, and S. Hong, "Resampling algorithms for particle filters: A computational complexity perspective," EURASIP Journal on Applied Signal Processing, vol. 2004, no. 15, pp. 22672277, 2004.

[26] M. S. Arulampalam, S. Maskell, N. Gordon, and T. Clapp, "A tutorial on particle filters for online nonlinear/non-gaussian bayesian tracking," IEEE Transactions on Signal Processing, vol. 50, no. 2, pp. 174-188, Feb. 2002. 